ESAIM: COCV 26 (2020) 50

https://doi.org/10.1051/cocv/2019023
ESAIM: Control, Optimisation and Calculus of Variations

www.esaim-cocv.org

\title{
ASYMPTOTIC LIMIT OF LINEAR PARABOLIC EQUATIONS WITH SPATIO-TEMPORAL DEGENERATED POTENTIALS
}

\author{
Pablo Àlvarez-Caudevilla, Matthieu Bonnivard \\ and Antoine Lemenant*
}

\begin{abstract}
In this paper, we observe how the heat equation in a noncylindrical domain can arise as the asymptotic limit of a parabolic problem in a cylindrical domain, by adding a potential that vanishes outside the limit domain. This can be seen as a parabolic version of a previous work by the first and last authors, concerning the stationary case [Alvarez-Caudevilla and Lemenant, Adv. Differ. Equ. 15 (2010) 649-688]. We provide a strong convergence result for the solution by use of energetic methods and $\Gamma$-convergence technics. Then, we establish an exponential decay estimate coming from an adaptation of an argument due to B. Simon.
\end{abstract}

Mathematics Subject Classification. 35A05, 35A15

Received April 9, 2018. Accepted April 7, 2019.

\section{INTRODUCTION}

For $\Omega \subset \mathbb{R}^{N}$ open and $T>0$, we define the cylinder $Q_{T}=\Omega \times(0, T)$. Let $\lambda>0$ be a positive real parameter. For $f_{\lambda} \in L^{2}\left(Q_{T}\right), g_{\lambda} \in H_{0}^{1}(\Omega)$ and $a: Q_{T} \rightarrow \mathbb{R}^{+}$a bounded measurable function, we consider the solution $u_{\lambda}$ of the parabolic problem

$$
\left(P_{\lambda}\right) \begin{cases}\partial_{t} u-\Delta u+\lambda a(x, t) u=f_{\lambda} & \text { in } Q_{T}, \\ u=0 & \text { on } \partial \Omega \times(0, T), \\ u(x, 0)=g_{\lambda}(x) & \text { in } \Omega .\end{cases}
$$

Since $\left(P_{\lambda}\right)$ is a classical parabolic problem, existence and regularity of solutions follow from standard theory well developed in the literature (see Sect. 3). In particular, under our assumptions, $u \in L^{2}\left(0, T ; H_{0}^{1}(\Omega)\right.$ ) is continuous in time with values in $L^{2}(\Omega)$ (thus the initial condition $u(x, 0)=g_{\lambda}(x)$ is well defined in $L^{2}(\Omega)$ ) and the equation is satisfied in a weak sense (see Sect. 3 for an exact formulation).

Keywords and phrases: Parabolic problems, Gamma-convergence, energetic methods, variational methods, partial differential equations.

Universite Paris Diderot, Paris, France.

* Corresponding author: lemenant@ljll.univ-paris-diderot.fr 
In this paper, we are interested in the limit of $u_{\lambda}$ when $\lambda \rightarrow+\infty$. In particular, we assume spatial and temporal degeneracies for the potential $a$, which means that

$$
O_{a}:=\operatorname{Int}\left(\left\{(x, t) \in Q_{T}: a(x, t)=0\right\}\right) \neq \emptyset .
$$

We also assume that $\partial O_{a}$ has zero Lebesgue measure.

In order to describe the results of this paper, let us start with elementary observations. Assume that, when $\lambda$ goes to $+\infty, f_{\lambda}$ converges to $f$ and $g_{\lambda}$ converges to $g$, for instance in $L^{2}$. Assume also that $u_{\lambda}$ converges weakly in $L^{2}\left(Q_{T}\right)$ to some $u \in L^{2}\left(Q_{T}\right)$.

Under those assumptions it is not very difficult to get the following a priori bound using the equation in $\left(P_{\lambda}\right)$ (see Lem. 5.1)

$$
\lambda \int_{Q_{T}} a u_{\lambda}^{2} \mathrm{~d} x \mathrm{~d} t \leq C
$$

This shows that $u_{\lambda}$ converges strongly to 0 in any set of the form $\{a(x, t)>\varepsilon\}$, for any $\varepsilon>0$.

Then, multiplying the equation in $\left(P_{\lambda}\right)$ by any $\varphi \in C_{0}^{\infty}\left(O_{a}\right)$ we get, after some integration by parts (in this paper we shall denote $\nabla$ for $\nabla_{x}$, i.e. the gradient in space),

$$
\int_{Q_{T}} u_{\lambda} \partial_{t} \varphi-\int_{Q_{T}} u_{\lambda} \Delta \varphi=\int_{Q_{T}} f_{\lambda} \varphi
$$

Passing to the limit, we obtain that $\partial_{t} u-\Delta u=f$ in $\mathcal{D}^{\prime}\left(O_{a}\right)$. Under some suitable extra assumptions on the potential $a$, we will actually be able to prove that the limit $u$ satisfies the following more precise problem:

$$
\left(P_{\infty}\right)\left\{\begin{array}{l}
u \in L^{2}\left(0, T ; H_{0}^{1}(\Omega)\right), \quad u^{\prime} \in L^{2}\left(0, T ; L^{2}(\Omega)\right) \\
u=0 \text { a.e. in } Q_{T} \backslash O_{a} \\
\int_{Q_{T}}\left(u^{\prime} v+\nabla u \nabla v\right)=\int_{Q_{T}} f v \\
\quad \text { for all } v \in L^{2}\left(0, T ; H_{0}^{1}(\Omega)\right) \text { s.t. } v=0 \text { a.e. in } Q_{T} \backslash O_{a} \\
u(x, 0)=g(x) \quad \text { in } \Omega .
\end{array}\right.
$$

Problem $\left(P_{\infty}\right)$, which arises here naturally as the limit problem associated with the family of problems $\left(P_{\lambda}\right)$, is a nonstandard Cauchy-Dirichlet problem for the heat equation since $O_{a}$ may, in general, not be cylindrical. This type of heat equation in a noncylindrical domain appears in many applications, and different approaches have been developed recently to solve problems related to $\left(P_{\infty}\right)$ (see for e.g. $[4-7,9,13]$ and the references therein). As a byproduct to our work, we have obtained an existence and uniqueness result for the problem $\left(P_{\infty}\right)$ (see Cor. 5.5).

Furthermore, in this paper we study in more detail the convergence of $u_{\lambda}$, when $\lambda$ goes to infinity. Our first result gives a sufficient condition on the potential $a$, for which the convergence of $u_{\lambda}$ to $u$ is stronger than a weak $L^{2}$ convergence. Indeed, assuming a monotonicity condition on the potential $a$, and using purely energetic and variational methods, we obtain that the convergence holds strongly in $L^{2}\left(0, T ; H^{1}(\Omega)\right)$; see Section 5 . Our approach can be seen as the continuation of a previous work [1], where the stationary problem was studied using the theory of $\Gamma$-convergence, as well as in [2] using a different analysis.

Here is our first main result. 
Theorem 1.1. For all $\lambda>0$, let $u_{\lambda}$ be the solution of $\left(P_{\lambda}\right)$ with $f_{\lambda} \in L^{2}(\Omega \times(0, T))$ and $g_{\lambda} \in H_{0}^{1}(\Omega)$. Assume that $a: \Omega \times[0, T] \rightarrow \mathbb{R}^{+}$is a Lipschitz function which satisfies

$$
\partial_{t} a(x, t) \leq 0 \text { a.e. in } Q_{T} \text {. }
$$

Assume also that the initial condition $g_{\lambda}$ satisfies

$$
\sup _{\lambda>0}\left(\lambda \int_{\Omega} a(x, 0) g_{\lambda}(x)^{2} \mathrm{~d} x\right)<+\infty
$$

converges weakly to $g$ in $L^{2}(\Omega)$, and that $f_{\lambda}$ converges weakly to $f$ in $L^{2}\left(Q_{T}\right)$.

Then $u_{\lambda}$ converges strongly to $u$ in $L^{2}\left(0, T ; H^{1}(\Omega)\right)$, where $u$ is the unique solution of $\left(P_{\infty}\right)$.

Remark 1.2. In particular, condition (1.3) implies that the family of sets $\Omega_{a}(t) \subset \Omega$, defined for $t>0$ by $\Omega_{a}(t):=\left\{x \in \Omega,(x, t) \in O_{a}\right\}$, is increasing in time for the inclusion. In that case, by a slight abuse of terminology, we will often write simply that $O_{a}$ is increasing in time (for the inclusion).

Our second result is a quantitative convergence of $u_{\lambda}$ to 0 , outside $O_{a}$ (in other words, away from the vanishing region), with very general assumptions on $a$ (only continuous and $O_{a} \neq \emptyset$ ), but in the special case when $f_{\lambda}=0$ in $Q_{T} \backslash O_{a}$. This is obtained using an adaptation of an argument due to Simon [14], and proves that $u_{\lambda}$ decays exponentially fast to 0 with respect to $\lambda$ in the region $Q_{T} \backslash O_{a}$. Compared to the standard bound (1.2), this results expresses that $u_{\lambda}$ goes to 0 much faster than one could expect. We also take the opportunity of this paper to write a similar estimate for the stationary problem (see Lem. 6.1 in Sect. 6).

Theorem 1.3. For all $\lambda>0$, let $u_{\lambda}$ be the solution of $\left(P_{\lambda}\right)$ with $f_{\lambda} \in L^{2}\left(Q_{T}\right)$ and $g_{\lambda} \in H_{0}^{1}\left(\overline{O_{a}} \cap\{t=0\}\right)$. Assume that $f_{\lambda}=0$ in $Q_{T} \backslash O_{a}$. Let $a: \bar{\Omega} \times[0, T] \rightarrow \mathbb{R}^{+}$be a continuous function for which $O_{a}$ is nonempty. For every $\varepsilon>0$, define $A_{\varepsilon}:=\left\{(x, t) ; \operatorname{dist}\left((x, t), O_{a}\right)>\varepsilon\right\}$. Then, for every $\varepsilon>0$, there exists a constant $C>0$ such that

$$
\sup _{\lambda>0}\left(\lambda e^{c_{\varepsilon} \sqrt{\lambda}} \int_{A_{\varepsilon}} u_{\lambda}^{2} \mathrm{~d} x\right) \leq C
$$

where $c_{\varepsilon}:=\varepsilon \min _{(x, t) \in A_{\varepsilon / 2}} a(x, t)$.

The convergence of weak solutions of $\left(P_{\lambda}\right)$ was already observed in [8] as a starting point for a more detailed analysis about the associated semigroup. This was then used in [8] to analyze the asymptotic behaviour of a nonlinear periodic-parabolic problem of logistic type (firstly analyzed by Hess [12]) where the equation is the following, also considered before in [9],

$$
\partial_{t} u-\Delta u=\mu u-a(x, t) u^{p}
$$

used in some models of population dynamics. A possible link between our problem $\left(P_{\lambda}\right)$ and the nonlinear equation (1.4) is coming from the fact that asymptotic limit of the principal eigenvalue for the linear parabolic operator $\partial_{t}-\Delta+\lambda a(x, t)$ plays a role in the dynamical behaviour of nonlinear logistic equation $(c f .[3,8,9,11])$. We thus believe that the results and techniques developed in the present paper could possibly be used in the study of more general equations such as (1.4).

Furthermore, another possible application of our results could be for numerical purposes. Indeed, for the ones who would be interested by computing a numerical solution of the noncylindrical limiting problem $\left(P_{\infty}\right)$, one could use the cylindrical problem $\left(P_{\lambda}\right)$ for a large $\lambda$, much easier to compute via standard methods. The strong 
convergence stated in Theorem 1.1, together with the exponential rate of convergence stated in Theorem 1.3, gives some good estimates about the difference between those two different solutions.

\section{The Stationary PROBlem}

This section concerns only the stationary problem. In particular, throughout the section, all functions $u, a, f$, etc., will be functions of $x \in \Omega$ (and independent of $t$ ).

We assume $\Omega \subset \mathbb{R}^{N}$ to be an open and bounded domain and $a: \bar{\Omega} \rightarrow \mathbb{R}^{+}$be a measurable and bounded non-negative function. We suppose that

$$
K_{a}:=\{x \in \bar{\Omega} ; a(x)=0\} \subset \Omega \text { is a closed set in } \mathbb{R}^{N} .
$$

Moreover, we assume that

$$
\Omega_{a}:=\operatorname{Int}\left(K_{a}\right) \neq \emptyset
$$

Under hypothesis (2.1) we know that

$$
H_{0}^{1}\left(K_{a}\right):=H^{1}\left(\mathbb{R}^{N}\right) \cap\left\{u=0 \text { q.e. in } \mathbb{R}^{N} \backslash K_{a}\right\}=H^{1}\left(\mathbb{R}^{N}\right) \cap\left\{u=0 \text { a.e. in } \mathbb{R}^{N} \backslash K_{a}\right\},
$$

and hypothesis (2.2) implies that

$$
H_{0}^{1}\left(K_{a}\right) \neq\{0\}
$$

Notice that we are working with a functional space of the form $H_{0}^{1}(A)$, where $A$ is a closed subset of $\mathbb{R}^{N}$. Therefore, we do not claim that $H_{0}^{1}(A)=H_{0}^{1}(\operatorname{Int}(A))$, which is true only under more regularity assumptions on the set $A$.

Furthermore, we define the functionals $E_{\lambda}$ and $E$ on $L^{2}(\Omega)$ as follows.

$$
\begin{aligned}
& E_{\lambda}(u)= \begin{cases}\int_{\Omega}|\nabla u|^{2}+\lambda a u^{2} \mathrm{~d} x & \text { if } u \in H_{0}^{1}(\Omega) \\
+\infty & \text { otherwise }\end{cases} \\
& E(u)= \begin{cases}\int_{\Omega}|\nabla u|^{2} \mathrm{~d} x & \text { if } u \in H_{0}^{1}\left(K_{a}\right) \\
+\infty & \text { otherwise }\end{cases}
\end{aligned}
$$

The following result was already stated and used in [1]. For the sake of completeness, we reproduce the proof here and refer the reader to [1] for the connection of this result with $\Gamma$-convergence and several examples.

Proposition 2.1. Let $f_{\lambda} \in L^{2}(\Omega)$ be a family of functions indexed by some real parameter $\lambda>0$ and uniformly bounded in $L^{2}(\Omega)$. Moreover, assume that $f_{\lambda}$ converges to a function $f \in L^{2}(\Omega)$ in the weak topology of $L^{2}(\Omega)$, when $\lambda$ tends to $+\infty$. Then the unique solution $u_{\lambda}$ of the problem

$$
\left(P_{\lambda}^{s}\right)\left\{\begin{array}{c}
-\Delta u_{\lambda}+\lambda a u_{\lambda}=f_{\lambda} \\
u_{\lambda} \in H_{0}^{1}(\Omega),
\end{array}\right.
$$


converges strongly in $H^{1}(\Omega)$, when $\lambda \rightarrow+\infty$, to the unique solution of the problem

$$
\left(P_{\infty}^{s}\right)\left\{\begin{array}{l}
-\Delta u=f \\
u \in H_{0}^{1}\left(K_{a}\right) .
\end{array}\right.
$$

Proof. This is a standard consequence of the $\Gamma$-convergence of energies $E_{\lambda}$, which relies on the fact that $u_{\lambda}$ is the unique minimizer in $H_{0}^{1}(\Omega)$ for

$$
v \mapsto E_{\lambda}(v)-2 \int_{\Omega} f_{\lambda} v
$$

whereas $u$ is the unique minimizer in $H_{0}^{1}\left(K_{a}\right)$ for

$$
v \mapsto E(v)-2 \int_{\Omega} f v
$$

Let us write the full details of the proof. For any $\lambda>0$, let $u_{\lambda}$ be the solution of $\left(P_{\lambda}^{s}\right)$. We first prove that $\left\{u_{\lambda}\right\}_{\lambda>0}$ is compact in $L^{2}(\Omega)$. This comes from the energy equality

$$
\int_{\Omega}\left(\left|\nabla u_{\lambda}\right|^{2}+\lambda a u_{\lambda}^{2}\right) \mathrm{d} x=\int_{\Omega} f_{\lambda} u_{\lambda} \mathrm{d} x
$$

which implies

$$
\int_{\Omega}\left|\nabla u_{\lambda}\right|^{2} \leq\left\|f_{\lambda}\right\|_{L^{2}(\Omega)}\left\|u_{\lambda}\right\|_{L^{2}(\Omega)} \leq C\left\|u_{\lambda}\right\|_{L^{2}(\Omega)} \quad \text { with } C \text { a positive constant. }
$$

Thanks to Poincaré's inequality we also have that

$$
\left\|u_{\lambda}\right\|_{L^{2}(\Omega)}^{2} \leq C(\Omega) \int_{\Omega}\left|\nabla u_{\lambda}\right|^{2} \mathrm{~d} x
$$

which finally proves that $u_{\lambda}$ is uniformly bounded in $H_{0}^{1}(\Omega)$.

Now let $w$ be any point in the $L^{2}$-adherence of the family $\left\{u_{\lambda}\right\}_{\lambda>0}$. In other words, there exists a subsequence, still denoted by $u_{\lambda}$, converging strongly in $L^{2}$ to $w$. Since $u_{\lambda}$ is bounded in $H^{1}(\Omega)$, we can assume, up to extracting a further subsequence, that $u_{\lambda}$ converges weakly in $H^{1}(\Omega)$ to a function that must necessarily be $w$.

Now let $u$ be the solution of the limit problem $\left(P_{\infty}^{s}\right)$. By definition of $\left(P_{\infty}^{s}\right), u \in H_{0}^{1}\left(K_{a}\right)$ and in particular $a u=0$ almost everywhere in $\Omega$ and $E_{\lambda}(u)=E(u)$ for all $\lambda>0$. Now since $u_{\lambda}$ is a minimizer of

$$
u \mapsto E_{\lambda}(u)-2 \int_{\Omega} f_{\lambda} u \mathrm{~d} x
$$

and $u$ is admissible, we have

$$
E_{\lambda}\left(u_{\lambda}\right)-2 \int_{\Omega} f_{\lambda} u_{\lambda} \mathrm{d} x \leq E_{\lambda}(u)-2 \int_{\Omega} f_{\lambda} u \mathrm{~d} x=E(u)-2 \int_{\Omega} f_{\lambda} u \mathrm{~d} x .
$$


Hence, letting $\lambda$ go to infinity in the previous inequality, using the trivial inequality $E\left(u_{\lambda}\right) \leq E_{\lambda}\left(u_{\lambda}\right)$ and then the lower-semicontinuity of the Dirichlet integral with respect to the weak convergence, it follows that

$$
\begin{aligned}
E(w)-2 \int_{\Omega} f w \mathrm{~d} x & \leq \liminf _{\lambda}\left(E_{\lambda}\left(u_{\lambda}\right)-2 \int_{\Omega} f_{\lambda} u_{\lambda} \mathrm{d} x\right) \\
& \leq \limsup _{\lambda}\left(E_{\lambda}\left(u_{\lambda}\right)-2 \int_{\Omega} f_{\lambda} u_{\lambda} \mathrm{d} x\right) \\
& \leq E(u)-2 \int_{\Omega} f u \mathrm{~d} x
\end{aligned}
$$

which shows that $w$ is a minimizer, and thus $w=u$. By uniqueness of the adherence point, we infer that the whole sequence $u_{\lambda}$ converges strongly in $L^{2}$ to $u$ (and weakly in $H^{1}$ ).

It remains to prove the strong convergence in $H^{1}$. To do so, it is enough to prove

$$
\left\|\nabla u_{\lambda}\right\|_{L^{2}(\Omega)} \rightarrow\|\nabla u\|_{L^{2}(\Omega)}
$$

Due to the weak convergence in $H^{1}(\Omega)$ (up to subsequences) we already have

$$
\|\nabla u\|_{L^{2}(\Omega)} \leq \liminf _{\lambda}\left\|\nabla u_{\lambda}\right\|_{L^{2}(\Omega)}
$$

and going back to (2.6) we get the reverse inequality, with a limsup.

The proof of convergence of the whole sequence follows by uniqueness of the adherent point in $L^{2}(\Omega)$.

Remark 2.2. Notice that when $u$ is a solution of $\left(P_{\infty}^{s}\right)$, then $-\Delta u=f$ only in $\operatorname{Int}\left(K_{a}\right)$ and $-\Delta u=0$ in $K_{a}^{c}$. However, in general $-\Delta u$ has a singular part on $\partial K_{a}$. Typically, if $K_{a}$ is for instance a set of finite perimeter, then in the distributional sense in $\Omega$,

$$
-\Delta u=f \mathbf{1}_{K_{a}}+\left.\frac{\partial u}{\partial \nu} \mathcal{H}^{N-1}\right|_{\partial K_{a}},
$$

where $\nu$ is the outer normal on $\partial K_{a}$ and $\mathcal{H}^{N-1}$ is the $N-1$ dimensional Hausdorff measure.

As a consequence of Proposition 2.1, we easily obtain the following result.

Proposition 2.3. Assume that $f_{\lambda}$ converges weakly to a function $f$ in $L^{2}(\Omega)$. For any $\lambda>0$, let $u_{\lambda}$ be the solution of problem $\left(P_{\lambda}^{s}\right)$. Then, when $\lambda \rightarrow \infty$,

$$
\begin{gathered}
\lambda \int_{\Omega} a u_{\lambda}^{2} \mathrm{~d} x \rightarrow 0, \\
\lambda a u_{\lambda} \rightarrow f \mathbf{1}_{\Omega \backslash K_{a}}+\left.(\Delta u)\right|_{\partial K_{a}} \text { in } \mathcal{D}^{\prime}(\Omega),
\end{gathered}
$$

where $u$ is the solution of $\left(P_{\infty}^{s}\right)$. Moreover, the convergence in $(2.8)$ holds in the weak-* topology of $H^{-1}$.

Proof. Due to Proposition 2.1 we know that $u_{\lambda}$ converges strongly in $H^{1}(\Omega)$ to $u$, the solution of problem $\left(P_{\infty}^{s}\right)$. In particular, from the fact that

$$
\int_{\Omega}\left|\nabla u_{\lambda}\right|^{2} \mathrm{~d} x \rightarrow \int_{\Omega}|\nabla u|^{2} \mathrm{~d} x=\int_{\Omega} u f \mathrm{~d} x
$$


and

$$
\int_{\Omega} u_{\lambda} f_{\lambda} \mathrm{d} x \rightarrow \int_{\Omega} u f \mathrm{~d} x
$$

passing to the limit in the following energy equality

$$
\int_{\Omega}\left|\nabla u_{\lambda}\right|^{2} \mathrm{~d} x+\lambda \int_{\Omega} a u_{\lambda}^{2} \mathrm{~d} x=\int_{\Omega} u_{\lambda} f_{\lambda} \mathrm{d} x
$$

we obtain (2.7). Next, let us now prove (2.8). Thus, since $u_{\lambda}$ is a solution of $\left(P_{\lambda}^{s}\right)$ then, for every test function $\psi \in C_{c}^{\infty}(\Omega)$, after integrating by parts in $\Omega$ we arrive at

$$
\int_{\Omega} u_{\lambda}(-\Delta \psi) \mathrm{d} x+\lambda \int_{\Omega} a u_{\lambda} \psi \mathrm{d} x=\int_{\Omega} f_{\lambda} \psi \mathrm{d} x
$$

Passing to the limit we obtain that $\lambda a u_{\lambda} \rightarrow f+\Delta u$ in $\mathcal{D}^{\prime}(\Omega)$. Now returning to (2.10), we can write, for every $\psi$ satisfying $\|\psi\|_{H^{1}(\Omega)} \leq 1$

$$
\left|\lambda \int_{\Omega} a u_{\lambda} \psi \mathrm{d} x\right| \leq\left\|f_{\lambda}\right\|_{2}+\left\|\nabla u_{\lambda}\right\|_{2} \leq C
$$

Taking the supremum in $\psi$ we get

$$
\left\|\lambda a u_{\lambda}\right\|_{H^{-1}} \leq C
$$

Therefore, $\lambda a u_{\lambda}$ is weakly-* sequentially compact in $H^{-1}$ and we obtain the convergence by uniqueness of the limit in the distributional sense.

\section{Existence AND REgularity of SOlutions For $\left(P_{\lambda}\right)$}

In order to define properly a solution for $\left(P_{\lambda}\right)$, we first recall the definition of the spaces $L^{p}(0, T ; X)$, with $X$ a Banach space, which consist of all (strongly) measurable functions (see [10], Appendix E.5) $u:[0, T] \rightarrow X$ such that

$$
\|u\|_{L^{p}(0, T ; X)}=\left(\int_{0}^{T}\|u(t)\|_{X}^{p} \mathrm{~d} t\right)^{1 / p}<+\infty
$$

for $1 \leq p<+\infty$, and

$$
\|u\|_{L^{\infty}(0, T ; X)}=\underset{t \in(0, T)}{\operatorname{ess} \sup }\|u(t)\|_{X}<+\infty .
$$

For simplicity we will sometimes use the following notation for $p=2$ and $X=L^{2}(\Omega)$ :

$$
\|\cdot\|_{2} \equiv\|\cdot\|_{L^{2}\left(0, T ; L^{2}(\Omega)\right)} \cdot
$$

We will also use the notation $u(x, t)=u(t)(x)$ for $(x, t) \in \Omega \times(0, T)$. 
Next, we will denote by $u^{\prime}$ the derivative of $u$ in the $t$ variable, intended in the following weak sense: we say that $u^{\prime}=v$, with $u, v \in L^{2}(0, T ; X)$ and

$$
\int_{0}^{T} \varphi^{\prime}(t) u(t) \mathrm{d} t=-\int_{0}^{T} \varphi(t) v(t) \mathrm{d} t
$$

for all scalar test functions $\varphi \in C_{0}^{\infty}(0, T)$. The space $H^{1}(0, T ; X)$ consists of all functions $u \in L^{2}(0, T ; X)$ such that $u^{\prime} \in L^{2}(0, T ; X)$.

We will often use the following remark.

Remark 3.1. By ([10], Thm. 3, p. 303), if $u \in L^{2}\left(0, T ; H_{0}^{1}(\Omega)\right)$ and $u^{\prime} \in L^{2}\left(0, T ; H^{-1}(\Omega)\right)$, then $u \in$ $C\left([0, T], L^{2}(\Omega)\right)$ (after possibly being redefined on a set of measure zero). Moreover, the mapping $t \mapsto\|u(t)\|_{L^{2}(\Omega)}^{2}$ is absolutely continuous and

$$
\frac{\mathrm{d}}{\mathrm{d} t}\|u(t)\|_{L^{2}(\Omega)}^{2}=2\left\langle u^{\prime}(t), u(t)\right\rangle_{L^{2}(\Omega)} .
$$

In this section, we collect some useful information about the solution $u_{\lambda}$ of problem $\left(P_{\lambda}\right)$ coming from the classical theory of parabolic problems that can be directly found in the literature.

Firstly, existence and uniqueness of a weak solution $u_{\lambda}$ for the problem $\left(P_{\lambda}\right)$ follows from the standard Galerkin method; see ([10], Thms. 3 and 4, Sect. 7.1) for further details. According to this theory, a weak solution means that:

$$
\left(P_{\lambda}\right)\left\{\begin{array}{l}
u \in L^{2}\left(0, T ; H_{0}^{1}(\Omega)\right), \quad u^{\prime} \in L^{2}\left(0, T ; H^{-1}(\Omega)\right) \\
\int_{0}^{T}\left\langle u^{\prime}(t), v(t)\right\rangle_{\left(H^{-1}, H_{0}^{1}\right)}+\int_{Q_{T}}(\nabla u \cdot \nabla v+\lambda a u v)=\int_{Q_{T}} f_{\lambda} v \\
\quad \text { for all } v \in L^{2}\left(0, T ; H_{0}^{1}(\Omega)\right), \\
u(0)=g_{\lambda}(x) \quad \text { in } L^{2}(\Omega) .
\end{array}\right.
$$

Remember that by Remark 3.1 above, such weak solution $u$ is continuous in time with values in $L^{2}(\Omega)$ so that the initial condition makes sense. In the rest of the paper, $\left(P_{\lambda}\right)$ will always refer to the above precise formulation of the problem that was first stated in Section 1.

Next, thanks to ([10], Thm. 5, Sect. 7.1), by considering $\lambda a u$ as a right hand side term (in $L^{2}(\Omega \times(0, T))$ ), we have the following.

Lemma 3.2. Let $\lambda>0, g_{\lambda} \in H_{0}^{1}(\Omega), f_{\lambda} \in L^{2}\left(0, T ; L^{2}(\Omega)\right)$, and let $u_{\lambda}$ be the weak solution to $\left(P_{\lambda}\right)$. Then,

$$
u_{\lambda} \in L^{2}\left(0, T ; H^{2}(\Omega)\right) \cap L^{\infty}\left(0, T ; H_{0}^{1}(\Omega)\right), \quad u_{\lambda}^{\prime} \in L^{2}\left(0, T ; L^{2}(\Omega)\right),
$$

and $u_{\lambda}$ satisfies the following estimate:

$$
\begin{gathered}
\sup _{0 \leq t \leq T}\left\|u_{\lambda}(t)\right\|_{H_{0}^{1}(\Omega)}+\left\|u_{\lambda}\right\|_{L^{2}\left(0, T ; H^{2}(\Omega)\right)}+\left\|u_{\lambda}^{\prime}\right\|_{2} \\
\leq C\left(\lambda\left\|a u_{\lambda}\right\|_{2}+\left\|f_{\lambda}\right\|_{2}+\left\|g_{\lambda}\right\|_{H_{0}^{1}(\Omega)}\right),
\end{gathered}
$$

where the constant $C$ depends only on $\Omega$ and $T$.

Remark 3.3. Notice that the bound (3.1) is not very useful when $\lambda \rightarrow+\infty$ since what we usually control is $\sqrt{\lambda}\|a u\|_{2}$ (shown below in Lem. 5.1) but not $\lambda\|a u\|_{2}$. Thus, the right hand side blows-up a priori. 


\section{UNiqueness OF SOLUTION FOR $\left(P_{\infty}\right)$}

In this section, we focus on the following problem that will arise as the limit of $\left(P_{\lambda}\right)$. Our notion of solution for the problem $\partial_{t} u-\Delta u=f$ in $O_{a}$ will precisely be the following:

$$
\left(P_{\infty}\right)\left\{\begin{array}{l}
u \in L^{2}\left(0, T ; H_{0}^{1}(\Omega)\right), \quad u^{\prime} \in L^{2}\left(0, T ; L^{2}(\Omega)\right) \\
u=0 \text { a.e. in } Q_{T} \backslash O_{a} \\
\int_{Q_{T}}\left(u^{\prime} v+\nabla u \cdot \nabla v\right)=\int_{Q_{T}} f v \\
\quad \text { for all } v \in L^{2}\left(0, T ; H_{0}^{1}(\Omega)\right) \text { s.t. } v=0 \text { a.e. in } Q_{T} \backslash O_{a} \\
u(0)=g(x) \quad \text { in } L^{2}(\Omega) .
\end{array}\right.
$$

As a byproduct of Section 5 we will prove the existence of a solution for the problem $\left(P_{\infty}\right)$, as a limit of solutions for $\left(P_{\lambda}\right)$. In this section, we prove the uniqueness which follows from a simple energy bound. Notice that a solution $u$ to $\left(P_{\infty}\right)$ is continuous in time (see Rem. 3.1) thus the initial condition $u(x, 0)=g(x)$ makes sense in $L^{2}(\Omega)$.

Proposition 4.1. Any solution $u$ of $\left(P_{\infty}\right)$ satisfies the following energy bound

$$
\frac{1}{4} \sup _{t \in(0, T)}\|u(t)\|_{L^{2}(\Omega)}^{2}+\|\nabla u\|_{2}^{2} \leq \frac{1}{2}\|g\|_{L^{2}(\Omega)}^{2}+T\|f\|_{2} .
$$

Consequently, there exists at most one solution to problem $\left(P_{\infty}\right)$.

Proof. Let $u$ be a solution to $\left(P_{\infty}\right)$, and $s \in(0, T)$. Choosing $v=u \mathbf{1}_{(0, s)}$ (where $\mathbf{1}_{(0, s)}$ is the characteristic function of $(0, s))$ in the weak formulation of the problem, we deduce that

$$
\int_{0}^{s} \int_{\Omega} u^{\prime} u \mathrm{~d} x \mathrm{~d} t+\int_{0}^{s} \int_{\Omega}|\nabla u|^{2} \mathrm{~d} x \mathrm{~d} t=\int_{0}^{s} \int_{\Omega} f u \mathrm{~d} x \mathrm{~d} t .
$$

Now applying Remark 3.1 and using the fact that $u \in L^{2}\left(0, T ; H_{0}^{1}(\Omega)\right)$ and $u^{\prime} \in L^{2}\left(0, T ; L^{2}(\Omega)\right)$ we obtain that $t \mapsto\|u(t)\|_{2}^{2}$ is absolutely continuous, and for a.e. $t$, there holds

$$
\frac{\mathrm{d}}{\mathrm{d} t}\|u(t)\|_{L^{2}(\Omega)}^{2}=2\left\langle u^{\prime}(t), u(t)\right\rangle_{L^{2}(\Omega)} .
$$

Returning to (4.2) we get

$$
\frac{1}{2}\|u(s)\|_{L^{2}(\Omega)}^{2}-\frac{1}{2}\|u(0)\|_{L^{2}(\Omega)}^{2}+\int_{0}^{s} \int_{\Omega}|\nabla u|^{2} \mathrm{~d} x \mathrm{~d} t=\int_{0}^{s} \int_{\Omega} f u \mathrm{~d} x \mathrm{~d} t .
$$

By Young's inequality we have

$$
\begin{aligned}
\left|\int_{0}^{s} \int_{\Omega} f u \mathrm{~d} x \mathrm{~d} t\right| & \leq \frac{\alpha}{2}\|f\|_{L^{2}(\Omega \times(0, s))}^{2}+\frac{1}{2 \alpha}\|u\|_{L^{2}(\Omega \times(0, s))}^{2} \\
& \leq \frac{\alpha}{2}\|f\|_{L^{2}(\Omega \times(0, s))}^{2}+\frac{T}{2 \alpha} \sup _{t \in(0, T)}\|u(t)\|_{L^{2}(\Omega)}^{2}
\end{aligned}
$$


Setting $\alpha=2 T$, estimating (4.3) by (4.4) and passing to the supremum in $s \in(0, T)$ finally gives

$$
\frac{1}{4} \sup _{t \in(0, T)}\|u(t)\|_{L^{2}(\Omega)}^{2}+\|\nabla u\|_{2}^{2} \leq \frac{1}{2}\|g\|_{L^{2}(\Omega)}^{2}+T\|f\|_{2}^{2},
$$

as desired.

Now assume that $u_{1}$ and $u_{2}$ are two solutions of $\left(P_{\infty}\right)$, and set $w:=u_{1}-u_{2}$. Then $w$ is a solution of $\left(P_{\infty}\right)$ with $f=0$ and $g=0$. Therefore, applying (4.1) to $w$ automatically gives $w=0$, which proves the uniqueness of the solution of $\left(P_{\infty}\right)$.

\section{Convergence of $u_{\lambda}$}

We now analyze the convergence of $u_{\lambda}$, which will follow from energy bounds for $u_{\lambda}$ and $u_{\lambda}^{\prime}$. As already mentioned before, the standard energy bound for the solutions of $\left(P_{\lambda}\right)$ that is stated in Lemma 3.2 blows up a priori when $\lambda$ goes to $+\infty$. Our goal in the sequel is to get better estimates, uniform in $\lambda$. The price to pay is the condition $\partial_{t} a \leq 0$ which implies that $O_{a}$ is nondecreasing in time (for the set inclusion).

\subsection{First energy bound}

Lemma 5.1. Assume that $g_{\lambda} \in L^{2}(\Omega)$ and $f_{\lambda} \in L^{2}\left(0, T ; L^{2}(\Omega)\right)$, and let $u_{\lambda}$ be the weak solution of problem $\left(P_{\lambda}\right)$. Then,

$$
\frac{1}{4} \sup _{t \in(0, T)}\left\|u_{\lambda}(t)\right\|_{L^{2}(\Omega)}^{2}+\left\|\nabla u_{\lambda}\right\|_{2}^{2}+\lambda \int_{0}^{T} \int_{\Omega} a u_{\lambda}^{2} \mathrm{~d} x \mathrm{~d} t \leq\left\|g_{\lambda}\right\|_{L^{2}(\Omega)}^{2}+T\left\|f_{\lambda}\right\|_{2}^{2} .
$$

Proof. Let $u_{\lambda}$ be a solution of $\left(P_{\lambda}\right)$ and $s \in(0, T)$. Testing with $v=u \mathbf{1}_{[0, s]}$ in the weak formulation of $\left(P_{\lambda}\right)$

$$
\begin{aligned}
& \frac{1}{2}\left\|u_{\lambda}(s)\right\|_{L^{2}(\Omega)}^{2}-\frac{1}{2}\left\|u_{\lambda}(0)\right\|_{L^{2}(\Omega)}^{2}+\int_{0}^{s} \int_{\Omega}\left|\nabla u_{\lambda}\right|^{2} \mathrm{~d} x \mathrm{~d} t+\lambda \int_{0}^{s} \int_{\Omega} a u_{\lambda}^{2} \mathrm{~d} x \mathrm{~d} t \\
& =\int_{0}^{s} \int_{\Omega} f_{\lambda} u_{\lambda} \mathrm{d} x \mathrm{~d} t .
\end{aligned}
$$

and arguing as in the proof of Proposition 4.1, we obtain (5.1), so that we omit the details.

\subsection{Second energy bound}

We now derive a uniform bound on $\left\|u_{\lambda}^{\prime}\right\|_{2}$. To this end, we will assume a time-monotonicity condition on $a$.

Definition 5.2 (Assumption (A)). We say that Assumption (A) hold if $a: Q_{T} \rightarrow \mathbb{R}^{+}$is Lipschitz and

$$
\partial_{t} a(x, t) \leq 0 \text { for a.e. }(x, t) \in Q_{T} .
$$

Lemma 5.3. We suppose that Assumption (A) holds. Then, the solution $u_{\lambda}$ of $\left(P_{\lambda}\right)$ satisfies the estimate:

$$
\begin{aligned}
& \int_{0}^{T} \int_{\Omega}\left(u_{\lambda}^{\prime}\right)^{2} \mathrm{~d} x \mathrm{~d} t+\sup _{s \in(0, T)}\left(\int_{\Omega}\left|\nabla u_{\lambda}(s)\right|^{2} \mathrm{~d} x\right) \\
& \quad \leq \int_{0}^{T} \int_{\Omega} f_{\lambda}^{2} \mathrm{~d} x \mathrm{~d} t+\int_{\Omega}\left|\nabla u_{\lambda}(0)\right|^{2} \mathrm{~d} x+\lambda \int_{\Omega} a(0) g_{\lambda}^{2} \mathrm{~d} x .
\end{aligned}
$$


Proof. Thanks to Lemma 3.2, we know that $u_{\lambda}^{\prime} \in L^{2}\left(0, T ; H_{0}^{1}(\Omega)\right)$. Consequently, for every $s \in(0, T)$, the function $v:=u_{\lambda}^{\prime} \mathbf{1}_{(0, s)}$ is an admissible test function in the weak formulation of $\left(P_{\lambda}\right)$. Hence, we obtain the identity

$$
\int_{0}^{s} \int_{\Omega}\left(u_{\lambda}^{\prime}\right)^{2} \mathrm{~d} x \mathrm{~d} t+\int_{0}^{s} \int_{\Omega} \nabla u_{\lambda} \cdot \nabla u_{\lambda}^{\prime} \mathrm{d} x \mathrm{~d} t+\lambda \int_{0}^{s} \int_{\Omega} a u_{\lambda} u_{\lambda}^{\prime} \mathrm{d} x \mathrm{~d} t=\int_{0}^{s} \int_{\Omega} f_{\lambda} u_{\lambda}^{\prime} \mathrm{d} x \mathrm{~d} t
$$

or written differently (applying Rem. 3.1),

$$
\begin{aligned}
\int_{0}^{s} & \int_{\Omega}\left(u_{\lambda}^{\prime}\right)^{2} \mathrm{~d} x \mathrm{~d} t+\int_{0}^{s}\left(\frac{1}{2} \int_{\Omega}\left|\nabla u_{\lambda}\right|^{2} \mathrm{~d} x\right)^{\prime} \mathrm{d} t+\lambda \int_{0}^{s}\left[\left(\frac{1}{2} \int_{\Omega} a u_{\lambda}^{2} \mathrm{~d} x\right)^{\prime}-\frac{1}{2} \int_{\Omega} a^{\prime} u_{\lambda}^{2} \mathrm{~d} x\right] \mathrm{d} t \\
& =\int_{0}^{s} \int_{\Omega} f_{\lambda} u_{\lambda}^{\prime} \mathrm{d} x \mathrm{~d} t .
\end{aligned}
$$

This yields

$$
\begin{gathered}
\int_{0}^{s} \int_{\Omega}\left(u_{\lambda}^{\prime}\right)^{2} \mathrm{~d} x \mathrm{~d} t+\frac{1}{2} \int_{\Omega}\left|\nabla u_{\lambda}(s)\right|^{2} \mathrm{~d} x+\frac{\lambda}{2} \int_{\Omega} a(s) u_{\lambda}(s)^{2} \mathrm{~d} x-\frac{\lambda}{2} \int_{0}^{s} \int_{\Omega} a^{\prime} u_{\lambda}^{2} \mathrm{~d} x \mathrm{~d} t \\
=\int_{0}^{s} \int_{\Omega} f_{\lambda} u_{\lambda}^{\prime} \mathrm{d} x \mathrm{~d} t+\frac{1}{2} \int_{\Omega}\left|\nabla u_{\lambda}(0)\right|^{2} \mathrm{~d} x+\frac{\lambda}{2} \int_{\Omega} a(0) u_{\lambda}(0)^{2} \mathrm{~d} x .
\end{gathered}
$$

By Young's inequality,

$$
\int_{0}^{s} \int_{\Omega} f_{\lambda} u_{\lambda}^{\prime} \mathrm{d} x \mathrm{~d} t \leq \frac{1}{2} \int_{0}^{s} \int_{\Omega} f_{\lambda}^{2} \mathrm{~d} x \mathrm{~d} t+\frac{1}{2} \int_{0}^{s} \int_{\Omega}\left(u_{\lambda}^{\prime}\right)^{2} \mathrm{~d} x \mathrm{~d} t
$$

so that we obtain

$$
\begin{gathered}
\int_{0}^{s} \int_{\Omega}\left(u_{\lambda}^{\prime}\right)^{2} \mathrm{~d} x \mathrm{~d} t+\int_{\Omega}\left|\nabla u_{\lambda}(s)\right|^{2} \mathrm{~d} x+\lambda \int_{\Omega} a(s) u_{\lambda}(s)^{2} \mathrm{~d} x-\lambda \int_{0}^{s} \int_{\Omega} a^{\prime} u_{\lambda}^{2} \mathrm{~d} x \mathrm{~d} t \\
\quad \leq \int_{0}^{s} \int_{\Omega}\left|f_{\lambda}\right|^{2} \mathrm{~d} x \mathrm{~d} t+\int_{\Omega}\left|\nabla u_{\lambda}(0)\right|^{2} \mathrm{~d} x+\lambda \int_{\Omega} a(0) u_{\lambda}(0)^{2} \mathrm{~d} x .
\end{gathered}
$$

Finally, using Assumption (A), the initial condition on $u_{\lambda}(0)$ and passing to the supremum in $s$, we conclude that estimate (5.3) holds.

\subsection{Weak convergence of solutions}

Using the previous energy estimates, we first establish the weak convergence of $u_{\lambda}$ to the solution $u$ of problem $\left(P_{\infty}\right)$, under Assumption $(A)$, and supposing certain bounds on the right hand side $f_{\lambda}$ and on the initial data $g_{\lambda}$.

Proposition 5.4. Assume that a satisfies Assumption (A). Let $\left\{f_{\lambda}\right\}$ be a bounded sequence in $L^{2}\left(Q_{T}\right)$ and $\left\{g_{\lambda}\right\}$ be a bounded sequence in $H_{0}^{1}(\Omega)$, satisfying

$$
\sup _{\lambda}\left(\lambda \int_{\Omega} a(0) g_{\lambda}^{2} \mathrm{~d} x\right)<\infty
$$


Up to extracting subsequences, we can assume that $f_{\lambda}$ converges weakly to a function $f$ in $L^{2}\left(Q_{T}\right)$, and $g_{\lambda}$ converges weakly to a function $g \in H_{0}^{1}(\Omega)$.

Let $u_{\lambda}$ be the solution of $\left(P_{\lambda}\right)$. Then $u_{\lambda}$ converges weakly in $L^{2}\left(Q_{T}\right)$ to the unique solution $u$ of problem $\left(P_{\infty}\right)$.

Proof. We know by Lemma 5.1 that $u_{\lambda}$ is uniformly bounded in $L^{2}\left(0, T ; H_{0}^{1}(\Omega)\right.$ ), thus converges weakly (up to extracting a subsequence) in $L^{2}\left(0, T ; H_{0}^{1}(\Omega)\right)$ to some function $u \in L^{2}\left(0, T ; H_{0}^{1}(\Omega)\right)$. Under Assumption $(A)$, we also know, thanks to Lemma 5.3, that

$$
\left\|u_{\lambda}^{\prime}\right\|_{L^{2}\left(Q_{T}\right)} \leq C
$$

so that, $u_{\lambda}^{\prime}$ also converges weakly in $L^{2}\left(Q_{T}\right)$ (up to extracting a further subsequence) to some limit $w \in L^{2}\left(Q_{T}\right)$, which must be equal to $u^{\prime}$ by uniqueness of the limit in $\mathcal{D}^{\prime}\left(Q_{T}\right)$. This shows that $u^{\prime} \in L^{2}\left(Q_{T}\right)$.

Next, due to (5.1) we know that

$$
\sup _{\lambda}\left(\lambda \int_{0}^{T} \int_{\Omega} a u_{\lambda}^{2} \mathrm{~d} x \mathrm{~d} t\right) \leq C
$$

which implies that, at the limit, $u$ must be equal to zero a.e. on any set of the form $\{a>\varepsilon\}$, with $\varepsilon>0$. By considering the union for $n \in \mathbb{N}^{*}$ of those sets with $\varepsilon=1 / n$, we obtain that $u=0$ a.e. on $Q_{T} \backslash O_{a}$.

Now let us check that $u$ satisfies the equation in the weak sense. Let $v$ be any test function in $L^{2}\left(0, T ; H_{0}^{1}(\Omega)\right)$ such that $v=0$ a.e. in $Q_{T} \backslash O_{a}$. Then $a u_{\lambda} v=0$ a.e. in $Q_{T}$, and using the fact that $u_{\lambda}$ is a solution of $\left(P_{\lambda}\right)$, we can write

$$
\left\langle u_{\lambda}^{\prime}, v\right\rangle_{L^{2}\left(Q_{T}\right)}+\left\langle\nabla u_{\lambda}, \nabla v\right\rangle_{L^{2}\left(Q_{T}\right)}=\left\langle f_{\lambda}, v\right\rangle_{L^{2}\left(Q_{T}\right)} .
$$

Thus, passing to the (weak) limit in $u_{\lambda}, u_{\lambda}^{\prime}$ and $f_{\lambda}$ we get

$$
\left\langle u^{\prime}, v\right\rangle_{L^{2}\left(Q_{T}\right)}+\langle\nabla u, \nabla v\rangle_{L^{2}\left(Q_{T}\right)}=\langle f, v\rangle_{L^{2}\left(Q_{T}\right)} .
$$

To conclude that $u$ is a solution of $\left(P_{\infty}\right)$ it remains to prove that $u(x, 0)=g(x)$ for a.e. $x \in \Omega$. For this purpose, we let $v \in C^{1}\left([0, T], H_{0}^{1}(\Omega)\right)$ be any function satisfying $v(T)=0$. Testing the equation with this $v$, using that $u_{\lambda}(0)=g_{\lambda}$ and integrating by parts with respect to $t$ we obtain

$$
-\left\langle g_{\lambda}, v(0)\right\rangle_{L^{2}(\Omega)}-\int_{0}^{T}\left\langle u_{\lambda}, v^{\prime}\right\rangle_{L^{2}(\Omega)}+\int_{0}^{T}\left\langle\nabla u_{\lambda}, \nabla v\right\rangle_{L^{2}(\Omega)}=\int_{0}^{T}\left\langle f_{\lambda}, v\right\rangle .
$$

Passing to the limit in $\lambda$ and using the weak convergence of $g_{\lambda}$ to $g$, we get

$$
-\langle g, v(0)\rangle_{L^{2}(\Omega)}-\int_{0}^{T}\left\langle u, v^{\prime}\right\rangle_{L^{2}(\Omega)}+\int_{0}^{T}\langle\nabla u, \nabla v\rangle_{L^{2}(\Omega)}=\int_{0}^{T}\langle f, v\rangle_{L^{2}(\Omega)} .
$$

Integrating back again by parts on $u$ yields

$$
\langle g, v(0)\rangle_{L^{2}(\Omega)}=\langle u(0), v(0)\rangle_{L^{2}(\Omega)},
$$

and since $v(0)$ is arbitrary, we deduce that $u(0)=g$ in $L^{2}(\Omega)$. 
Finally, the convergence of $u_{\lambda}$ to $u$ holds a priori up to a subsequence, but by uniqueness of the solution for the problem $\left(P_{\infty}\right)$ (see Prop. 4.1), the convergence holds for the whole sequence.

Corollary 5.5. Let $O_{a} \subset Q_{T}$ be open and increasing in time (in the sense of Rem. 1.2), and let $f \in L^{2}(\Omega \times$ $(0, T))$ and $g \in H_{0}^{1}(\Omega)$. Then there exists a (unique) solution for $\left(P_{\infty}\right)$.

Proof. It suffices to apply Proposition 5.4 with, for instance $a(x, t):=\operatorname{dist}\left((x, t), \overline{O_{a}}\right), f_{\lambda}=f$ and $g_{\lambda}=g$.

Remark 5.6. (Convergence in $\mathcal{D}^{\prime}(\Omega \times(0, T))$ ). Under Assumption $(A)$, letting $u$ being the weak limit of $u_{\lambda}$ in $L^{2}\left(Q_{T}\right)$, we already know that

$$
u=0 \quad \text { a.e. in } Q_{T} \backslash O_{a} .
$$

Then

$$
f_{\lambda}+\Delta u_{\lambda}-u_{\lambda}^{\prime} \longrightarrow f+\Delta u-u^{\prime} \text { in } \mathcal{D}^{\prime}(\Omega \times(0, T)),
$$

which implies that

$$
\lambda a u_{\lambda} \longrightarrow h \text { in } \mathcal{D}^{\prime}(\Omega \times(0, T)),
$$

for some distribution $h=f+\Delta u-u^{\prime} \in \mathcal{D}^{\prime}(\Omega \times(0, T))$, supported in $O_{a}^{c}$. Actually, since $u=0$ in $O_{a}^{c}$, we have

$$
\Delta u=0 \quad \text { and } \quad u^{\prime}=0 \quad \text { in } \mathcal{D}^{\prime}\left({\overline{O_{a}}}^{c}\right) .
$$

This means that

$$
h=0 \quad \text { in } \mathcal{D}^{\prime}\left(O_{a}\right) \quad \text { and } \quad h=f \text { in } \mathcal{D}^{\prime}\left({\overline{O_{a}}}^{c}\right) .
$$

Notice that, a priori, $h$ could have a singular part supported on $\partial O_{a}$. We finally deduce that

$$
\lambda a u_{\lambda} \underset{\lambda \rightarrow+\infty}{\longrightarrow} f \mathbf{1}_{O_{a}^{c}}+\left.\Delta u\right|_{\partial O_{a}} \text { in } \mathcal{D}^{\prime}(\Omega \times(0, T)) .
$$

\subsection{Strong convergence in $L^{2}\left(0, T ; H^{1}(\Omega)\right)$}

We now go further using the same argument as for the stationary problem, and prove a stronger convergence which is one of our main results.

Theorem 5.7. Under the same hypotheses as in Proposition 5.4, denote by $u_{\lambda}$ the solution of $\left(P_{\lambda}\right)$. Then, $u_{\lambda}$ converges strongly in $L^{2}\left(0, T ; H_{0}^{1}(\Omega)\right)$ to the solution $u$ of problem $\left(P_{\infty}\right)$.

Proof. We already have the bound

$$
\left\|u_{\lambda}\right\|_{L^{2}\left(0, T ; H^{1}(\Omega)\right)} \leq C,
$$

and we already know (by Prop. 5.4) that $u_{\lambda}$ converges weakly in $L^{2}\left(0, T ; H^{1}(\Omega)\right)$ to $u$, the unique solution of problem $\left(P_{\infty}\right)$.

Moreover, by the lower semicontinuity of the norm with respect to the weak convergence, there holds

$$
\|u\|_{L^{2}\left(0, T ; H^{1}(\Omega)\right)} \leq \liminf _{\lambda}\left\|u_{\lambda}\right\|_{L^{2}\left(0, T ; H^{1}(\Omega)\right)}
$$


Hence, to prove the strong convergence we only need to prove the reverse inequality, with a limsup. For this purpose we use the fact that $u(t)$ is a competitor for $u_{\lambda}(t)$ in the minimization problem solved by $u_{\lambda}$ at $t$ fixed. Indeed, for a.e. $t$ fixed, $u_{\lambda}$ solves

$$
-\Delta u_{\lambda}+\lambda a u_{\lambda}=f_{\lambda}-u_{\lambda}^{\prime}
$$

thus, $u_{\lambda}$ is a minimizer in $H_{0}^{1}(\Omega)$ for the energy

$$
v \mapsto E_{\lambda}(v)-2 \int_{\Omega} v\left(f_{\lambda}-u_{\lambda}^{\prime}\right)
$$

where $E_{\lambda}$ is defined by (2.3). Furthermore, due to the bound (5.3) obtained in Lemma 5.3, since $f_{\lambda}$ is bounded in $L^{2}\left(Q_{T}\right)$ and $g_{\lambda}$ is bounded in $L^{2}(\Omega)$ and satisfies (5.4), we know that $u_{\lambda}^{\prime}$ is bounded in $L^{2}\left(Q_{T}\right)$, and

$$
u_{\lambda}^{\prime} \rightarrow u^{\prime} \quad \text { weakly in } L^{2}\left(Q_{T}\right) .
$$

We also know that, up to a subsequence, $u_{\lambda} \rightarrow u$ strongly in $L^{2}\left(Q_{T}\right)$ (because it is bounded in $H^{1}\left(Q_{T}\right)$ ).

Now, using that $u$ is a competitor for $u_{\lambda}$ (for a.e. $t$ fixed), we obtain

$$
\begin{aligned}
\int_{\Omega}\left|\nabla u_{\lambda}\right|^{2} \mathrm{~d} x-2 \int_{\Omega} u_{\lambda}\left(f_{\lambda}-u_{\lambda}^{\prime}\right) & \leq E_{\lambda}\left(u_{\lambda}\right)-2 \int_{\Omega} u_{\lambda}\left(f_{\lambda}-u_{\lambda}^{\prime}\right) \leq E_{\lambda}(u)-2 \int_{\Omega} u\left(f_{\lambda}-u_{\lambda}^{\prime}\right) \\
& =\int_{\Omega}|\nabla u|^{2} \mathrm{~d} x-2 \int_{\Omega} u\left(f_{\lambda}-u_{\lambda}^{\prime}\right) .
\end{aligned}
$$

Integrating in $t \in[0, T]$, passing to the limsup in $\lambda$ and since we have the convergence

$$
\int_{Q_{T}} u_{\lambda}\left(f_{\lambda}-u_{\lambda}^{\prime}\right) \rightarrow \int_{Q_{T}} u\left(f-u^{\prime}\right)
$$

we get the desired inequality, which achieves the proof.

\section{Simon's EXPONEntial ESTimate}

\subsection{The stationary case}

Following a similar argument to ([14], Thm. 4.1) we ascertain some strong convergence far from the set $\Omega_{a}:=\operatorname{Int}\left(K_{a}\right)$, where $K_{a}$ is defined by $\left.K_{a}:=\{x \in \bar{\Omega} ; a(x)=0\}\right)$.

Lemma 6.1. Let $a: \bar{\Omega} \rightarrow \mathbb{R}^{+}$be a continuous non-negative potential and $u_{\lambda}$ be the unique weak solution in $H_{0}^{1}(\Omega)$ of $-\Delta u_{\lambda}+\lambda a u_{\lambda}=f_{\lambda}$ in $\Omega$. Assume that $\Omega_{a}:=\operatorname{Int}\{a(x)=0\}=\operatorname{Int}\left\{K_{a}\right\}$ is nonempty (hypothesis (2.2)). Let $\varepsilon>0$ be fixed, and define

$$
\Omega_{\varepsilon}:=\left\{x \in \Omega ; \operatorname{dist}\left(x, \Omega_{a}\right)>\varepsilon\right\} \quad \text { and } \quad \delta:=\min _{x \in \bar{\Omega}_{\varepsilon}} a(x)>0 .
$$

Then, there exists a constant $C>0$ such that for all $\lambda>0$ and for every $W^{2, \infty}$ function $\eta: \Omega \rightarrow \mathbb{R}$ that is equal to 1 in $\Omega_{2 \varepsilon}$ and to 0 outside $\Omega_{\varepsilon}$, we have

$$
\int_{\Omega_{\varepsilon}} e^{2 \sqrt{\lambda \frac{\delta}{2}} \operatorname{dist}\left(x, \Omega_{2 \varepsilon}^{c}\right)} \eta^{2} u_{\lambda}\left(\frac{\lambda \delta}{2} u_{\lambda}-f_{\lambda}\right) \mathrm{d} x \leq C
$$


with $C=C\left(\|\nabla \eta\|_{\infty},\|\Delta \eta\|_{\infty}, \varepsilon, \sup _{\lambda}\left\|f_{\lambda}\right\|_{2}\right)$.

Proof. Let $\varepsilon>0$ be fixed. For any function $\psi \in H_{0}^{1}\left(\Omega_{\varepsilon}\right)$ and for any function $\rho$ Lipschitz satisfying $|\nabla \rho|^{2} \leq \delta / 2$, we start by computing the integral

$$
\begin{aligned}
\int_{\Omega} & \nabla\left(e^{\sqrt{\lambda} \rho} \psi\right) \cdot \nabla\left(e^{-\sqrt{\lambda} \rho} \psi\right) \mathrm{d} x \\
& =\int_{\Omega}\left(\sqrt{\lambda} e^{\sqrt{\lambda} \rho} \psi \nabla \rho+e^{\sqrt{\lambda} \rho} \nabla \psi\right) \cdot\left(-\sqrt{\lambda} e^{-\sqrt{\lambda} \rho} \psi \nabla \rho+e^{-\sqrt{\lambda} \rho} \nabla \psi\right) \mathrm{d} x \\
& =\int_{\Omega}\left(-\lambda \psi^{2}|\nabla \rho|^{2}+|\nabla \psi|^{2}\right) \mathrm{d} x .
\end{aligned}
$$

As a result, there holds the estimate

$$
\begin{aligned}
\int_{\Omega}\left(\nabla\left(e^{\sqrt{\lambda} \rho} \psi\right) \cdot \nabla\left(e^{-\sqrt{\lambda} \rho} \psi\right)+\lambda a \psi^{2}\right) \mathrm{d} x & \geq \int_{\Omega} \lambda\left(a-|\nabla \rho|^{2}\right) \psi^{2} \mathrm{~d} x \\
& \geq \frac{\lambda \delta}{2} \int_{\Omega} \psi^{2} \mathrm{~d} x
\end{aligned}
$$

by definition of $\delta$.

Next, we apply (6.2) with the choice $\psi=e^{\sqrt{\lambda} \rho} \eta u_{\lambda}$, where $\eta \in W^{2, \infty}(\Omega, \mathbb{R})$ is equal to 1 in $\Omega_{2 \varepsilon}$ and equal to 0 outside $\Omega_{\varepsilon}$. Thus, using the following computation:

$$
\begin{aligned}
\nabla\left(e^{2 \sqrt{\lambda} \rho} \eta^{2} u_{\lambda}\right) \cdot \nabla u_{\lambda} & =\left[\nabla\left(e^{2 \sqrt{\lambda} \rho} \eta u_{\lambda}\right)+e^{2 \sqrt{\lambda} \rho} u_{\lambda} \nabla \eta\right] \cdot \eta \nabla u_{\lambda} \\
& =\nabla\left(e^{2 \sqrt{\lambda} \rho} \eta u_{\lambda}\right) \cdot\left(\nabla\left(\eta u_{\lambda}\right)-u_{\lambda} \nabla \eta\right)+e^{2 \sqrt{\lambda} \rho} u_{\lambda} \eta \nabla \eta \cdot \nabla u_{\lambda},
\end{aligned}
$$

and the fact that $\nabla \eta=0$ in $\Omega_{2 \varepsilon}$, we arrive at the following expression of the left-hand side of the previous inequality (6.2):

$$
\begin{aligned}
\int_{\Omega} & \left(\nabla\left(e^{\sqrt{\lambda} \rho} \psi\right) \cdot \nabla\left(e^{-\sqrt{\lambda} \rho} \psi\right)+\lambda a \psi^{2}\right) \mathrm{d} x \\
= & \int_{\Omega_{\varepsilon}}\left(\nabla\left(e^{2 \sqrt{\lambda} \rho} \eta u_{\lambda}\right) \cdot \nabla\left(\eta u_{\lambda}\right)+\lambda a e^{2 \sqrt{\lambda} \rho} \eta^{2} u_{\lambda}^{2}\right) \mathrm{d} x \\
= & \int_{\Omega_{\varepsilon}}\left(\nabla\left(e^{2 \sqrt{\lambda} \rho} \eta^{2} u_{\lambda}\right) \cdot \nabla u_{\lambda}+\lambda a e^{2 \sqrt{\lambda} \rho} \eta^{2} u_{\lambda}^{2}\right) \mathrm{d} x \\
& -\int_{\Omega_{\varepsilon} \backslash \overline{\Omega_{2 \varepsilon}}} e^{2 \sqrt{\lambda} \rho} u_{\lambda} \eta \nabla \eta \cdot \nabla u_{\lambda} \mathrm{d} x+\int_{\Omega_{\varepsilon} \backslash \overline{\Omega_{2 \varepsilon}}} \nabla\left(e^{2 \sqrt{\lambda} \rho} \eta u_{\lambda}\right) u_{\lambda} \nabla \eta \mathrm{d} x .
\end{aligned}
$$

Since the function $e^{2 \sqrt{\lambda} \rho} \eta^{2} u_{\lambda} \in H_{0}^{1}\left(\Omega_{\varepsilon}\right)$, it is an admissible test function for the equation satisfied by $u_{\lambda}$, it follows that

$$
\int_{\Omega_{\varepsilon}}\left(\nabla\left(e^{2 \sqrt{\lambda} \rho} \eta^{2} u_{\lambda}\right) \cdot \nabla u_{\lambda}+\lambda a e^{2 \sqrt{\lambda} \rho} \eta^{2} u_{\lambda}^{2}\right) \mathrm{d} x=\int_{\Omega_{\varepsilon}} e^{2 \sqrt{\lambda} \rho} \eta^{2} u_{\lambda} f_{\lambda} \mathrm{d} x
$$


Moreover, $u_{\lambda}$ satisfies $\left\|u_{\lambda}\right\|_{L^{2}(\Omega)}+\left\|\nabla u_{\lambda}\right\|_{L^{2}(\Omega)} \leq C\left\|f_{\lambda}\right\|_{L^{2}(\Omega)}$ for a constant $C$, so that by Young inequality,

$$
\left|\int_{\Omega_{\varepsilon} \backslash \overline{\Omega_{2 \varepsilon}}} e^{2 \sqrt{\lambda} \rho} u_{\lambda} \eta \nabla \eta \cdot \nabla u_{\lambda} \mathrm{d} x\right| \leq C\|\nabla \eta\|_{\infty}\left\|f_{\lambda}\right\|_{L^{2}(\Omega)}^{2} e^{2 \sqrt{\lambda} M}
$$

where $M$ is defined by

$$
M:=\sup _{x \in \Omega \backslash \overline{\Omega_{2 \varepsilon}}} \rho(x) .
$$

Finally, since $u_{\lambda} \nabla \eta \in H_{0}^{1}\left(\Omega_{\varepsilon} \backslash \overline{\Omega_{2 \varepsilon}}\right)$, we can apply an integration by parts to obtain

$$
\int_{\Omega_{\varepsilon} \backslash \overline{\Omega_{2 \varepsilon}}} \nabla\left(e^{2 \sqrt{\lambda} \rho} \eta u_{\lambda}\right) u_{\lambda} \nabla \eta \mathrm{d} x=-\int_{\Omega_{\varepsilon} \backslash \overline{\Omega_{2 \varepsilon}}} e^{2 \sqrt{\lambda} \rho} \eta u_{\lambda}\left(u_{\lambda} \Delta \eta+\nabla u_{\lambda} \cdot \nabla \eta\right) \mathrm{d} x .
$$

By a similar argument, we deduce the estimate

$$
\left|\int_{\Omega_{\varepsilon} \backslash \overline{\Omega_{2 \varepsilon}}} \nabla\left(e^{2 \sqrt{\lambda} \rho} \eta u_{\lambda}\right) u_{\lambda} \nabla \eta \mathrm{d} x\right| \leq C\left(\|\nabla \eta\|_{\infty}+\|\Delta \eta\|_{\infty}\right)\left\|f_{\lambda}\right\|_{L^{2}(\Omega)}^{2} e^{2 \sqrt{\lambda} M} .
$$

Gathering the previous estimates, we conclude that

$$
\frac{\lambda \delta}{2} \int_{\Omega_{\varepsilon}} e^{2 \sqrt{\lambda} \rho} \eta^{2} u_{\lambda}^{2} \mathrm{~d} x \leq \int_{\Omega_{\varepsilon}} e^{2 \sqrt{\lambda} \rho} \eta^{2} u_{\lambda} f_{\lambda} \mathrm{d} x+C\left\|f_{\lambda}\right\|_{L^{2}(\Omega)}^{2} e^{2 \sqrt{\lambda} M},
$$

where $C=C\left(\|\nabla \eta\|_{\infty},\|\Delta \eta\|_{\infty}, \varepsilon\right)$.

Now, we specify the function $\rho$ by setting

$$
\rho(x):=\sqrt{\frac{\delta}{2}} \operatorname{dist}\left(x, \Omega_{2 \varepsilon}^{c}\right),
$$

which satisfies all our needed assumptions (i.e. $\rho$ is Lipschitz with $|\nabla \rho|^{2} \leq \delta / 2$ and $\rho=0$ outside $\Omega_{\varepsilon}$ ). In this case, $M=0$ thus (6.3) simply implies

$$
\frac{\lambda \delta}{2} \int_{\Omega_{\varepsilon}} e^{2 \sqrt{\lambda} \rho} \eta^{2} u_{\lambda}^{2} \mathrm{~d} x \leq \int_{\Omega_{\varepsilon}} e^{2 \sqrt{\lambda} \rho} \eta^{2} u_{\lambda} f_{\lambda} \mathrm{d} x+C\left\|f_{\lambda}\right\|_{L^{2}(\Omega)}^{2},
$$

or differently,

$$
\int_{\Omega_{\varepsilon}} e^{2 \sqrt{\lambda} \rho} \eta^{2} u_{\lambda}\left(\frac{\lambda \delta}{2} u_{\lambda}-f_{\lambda}\right) \mathrm{d} x \leq C\left\|f_{\lambda}\right\|_{L^{2}(\Omega)}^{2}
$$

which ends the proof.

Remark 6.2. The previous lemma can be used for instance in the following two particular cases: first in the particular case when $f=0$ in $\Omega \backslash \Omega_{a}$. Thus, we get the useful rate of convergence of $u_{\lambda} \rightarrow 0$ as $\lambda \rightarrow 0$ far 
from $\Omega_{a}$ :

$$
\int_{\Omega_{2 \varepsilon}} \lambda e^{2 \sqrt{\lambda \frac{\delta}{2}} \operatorname{dist}\left(x, \Omega_{2 \varepsilon}^{c}\right)} u_{\lambda}^{2} \mathrm{~d} x \leq C
$$

This is much better compared to the usual and simple energy bound:

$$
\lambda \int_{\Omega} a u_{\lambda}^{2} \leq C
$$

Another application is when $u_{\lambda}$ is an eigenfunction (this is actually the original framework of Simon [14]), i.e. when $f_{\lambda}=\sigma(\lambda) u_{\lambda}$ and with $\sigma(\lambda)$ standing for the first eigenvalue associated with $u_{\lambda}$. In this case, since we are assuming that the potential $a$ might vanish in a subdomain (it could vanish at a single point, as performed by Simon [14]), we have that $\sigma(\lambda)$ is bounded ( $c f$. [2] for further details). Consequently, thanks to this bound for $\lambda$ large enough $\frac{\lambda \delta}{2}-\sigma(\lambda) \geq 1$ which implies

$$
\int_{\Omega_{2 \varepsilon}} e^{2 \sqrt{\lambda \frac{\delta}{2}} \operatorname{dist}\left(x, \Omega_{2 \varepsilon}^{c}\right)} u_{\lambda}^{2} \mathrm{~d} x \leq C
$$

\subsection{The parabolic case}

We now extend the previous decay estimate to the parabolic problem.

Lemma 6.3. Let $a: \bar{Q}_{T} \rightarrow \mathbb{R}^{+}$be a continuous non-negative potential such that $O_{a}$ is nonempty, $f_{\lambda} \in L^{2}\left(Q_{T}\right)$, $g_{\lambda} \in H_{0}^{1}\left(\overline{O_{a}} \cap\{t=0\}\right)$ and let $u_{\lambda}$ be the solution of $\left(P_{\lambda}\right)$.

For every $\varepsilon>0$, we define

$$
A_{\varepsilon}:=\left\{(x, t) \in \bar{Q}_{T} ; \operatorname{dist}\left((x, t), O_{a}\right)>\varepsilon\right\} \quad \text { and } \quad \delta:=\min _{(x, t) \in \overline{A_{\varepsilon}}} a(x, t)>0 .
$$

Then, for any $\lambda \geq 4$, and for any $W^{2, \infty}$ function $\eta: Q_{T} \rightarrow \mathbb{R}$ equal to 1 in $A_{2 \varepsilon}$ and 0 outside $A_{\varepsilon}$, there exists a constant $C>0$ such that

$$
\int_{A_{\varepsilon}} e^{\sqrt{\lambda} c_{\delta} \operatorname{dist}\left((x, t), A_{2 \varepsilon}^{c}\right)} \eta^{2} u_{\lambda}(x)\left(\frac{\lambda \delta}{2} u_{\lambda}-f_{\lambda}\right) \mathrm{d} x \mathrm{~d} t \leq C,
$$

with $c_{\delta}:=2 \min \left(\sqrt{\frac{\delta}{2}}, \frac{\delta}{2}\right)$ and $C=C\left(\varepsilon,\|\nabla \eta\|_{\infty},\|\Delta \eta\|_{\infty},\left\|\partial_{t} \eta\right\|_{\infty}, \sup _{\lambda}\left\|f_{\lambda}\right\|_{2}, \sup _{\lambda}\left\|g_{\lambda}\right\|_{2}\right)$.

Proof. Let $\varepsilon>0$ be fixed.

We consider any function $\psi \in L^{2}\left(0, T ; H_{0}^{1}(\Omega)\right)$ such that $\psi=0$ outside $A_{\varepsilon}$, and any Lipschitz function $\rho: Q_{T} \rightarrow \mathbb{R}$ satisfying

$$
\max \left(\left|\partial_{t} \rho\right|,|\nabla \rho|^{2}\right) \leq \delta / 2
$$

Integrating in time estimate (6.2), and using the definition of $\delta$, we obtain

$$
\int_{Q_{T}}\left(\nabla\left(e^{\sqrt{\lambda} \rho} \psi\right) \cdot \nabla\left(e^{-\sqrt{\lambda} \rho} \psi\right)+\lambda a \psi^{2}\right) \mathrm{d} x \mathrm{~d} t \geq \frac{\lambda \delta}{2} \int_{Q_{T}} \psi^{2} \mathrm{~d} x \mathrm{~d} t .
$$


Developing the derivative in time and using estimate (6.5), we get

$$
\begin{aligned}
\int_{Q_{T}} e^{\sqrt{\lambda} \rho} \psi \partial_{t}\left(e^{-\sqrt{\lambda} \rho} \psi\right) \mathrm{d} x \mathrm{~d} t & =-\sqrt{\lambda} \int_{Q_{T}} \psi^{2} \partial_{t} \rho \mathrm{d} x \mathrm{~d} t+\int_{Q_{T}} \psi \partial_{t} \psi \mathrm{d} x \mathrm{~d} t \\
& =-\sqrt{\lambda} \int_{Q_{T}} \psi^{2} \partial_{t} \rho \mathrm{d} x \mathrm{~d} t+\frac{1}{2}\left(\int_{\Omega} \psi(T)^{2} \mathrm{~d} x-\int_{\Omega} \psi(0)^{2} \mathrm{~d} x\right) \\
& \geq-\frac{\sqrt{\lambda} \delta}{2} \int_{Q_{T}} \psi^{2} \mathrm{~d} x \mathrm{~d} t-\frac{1}{2} \int_{\Omega} \psi(0)^{2} \mathrm{~d} x
\end{aligned}
$$

Gathering the previous estimates, we obtain

$$
\begin{aligned}
& \int_{Q_{T}}\left[e^{\sqrt{\lambda} \rho} \psi\left(\partial_{t}\left(e^{-\sqrt{\lambda} \rho} \psi\right)+\lambda a e^{-\sqrt{\lambda} \rho} \psi\right)+\nabla\left(e^{\sqrt{\lambda} \rho} \psi\right) \cdot \nabla\left(e^{-\sqrt{\lambda} \rho} \psi\right)\right] \mathrm{d} x \mathrm{~d} t \\
& \quad \geq \frac{\delta}{2}(\lambda-\sqrt{\lambda}) \int_{Q_{T}} \psi^{2} \mathrm{~d} x \mathrm{~d} t-\frac{1}{2} \int_{\Omega} \psi(0)^{2} \mathrm{~d} x .
\end{aligned}
$$

Next, we define $\psi=e^{\sqrt{\lambda} \rho} \eta u_{\lambda}$, where $\eta \in W^{2, \infty}\left(Q_{T}\right)$ is equal to 1 in $A_{2 \varepsilon}$ and 0 outside $A_{\varepsilon}$. We assume that $\lambda \geq 4$ so that $\lambda-\sqrt{\lambda} \geq \lambda / 2$. Due to the assumptions, $g \in H_{0}^{1}\left(\overline{O_{a}} \cap\{t=0\}\right)$ and, then, $\|\psi(0)\|_{L^{2}(\Omega)}=0$. Thus, (6.6) implies

$$
\begin{aligned}
& \frac{\lambda \delta}{4} \int_{Q_{T}} e^{2 \sqrt{\lambda} \rho} \eta^{2} u_{\lambda}^{2} \mathrm{~d} x \mathrm{~d} t \\
& \quad \leq \int_{Q_{T}}\left[\nabla\left(e^{2 \sqrt{\lambda} \rho} \eta u_{\lambda}\right) \cdot \nabla\left(\eta u_{\lambda}\right)+\lambda a e^{2 \sqrt{\lambda} \rho} \eta^{2} u_{\lambda}^{2}+e^{2 \sqrt{\lambda} \rho} \eta u_{\lambda} \partial_{t}\left(\eta u_{\lambda}\right)\right] \mathrm{d} x \mathrm{~d} t .
\end{aligned}
$$

Proceeding similarly as in the stationary case, we obtain the analogous expression

$$
\begin{aligned}
\int_{Q_{T}} & {\left[\nabla\left(e^{2 \sqrt{\lambda} \rho} \eta u_{\lambda}\right) \cdot \nabla\left(\eta u_{\lambda}\right)+\lambda a e^{2 \sqrt{\lambda} \rho} \eta^{2} u_{\lambda}^{2}\right] \mathrm{d} x \mathrm{~d} t } \\
= & \int_{A_{\varepsilon}}\left(\nabla\left(e^{2 \sqrt{\lambda} \rho} \eta^{2} u_{\lambda}\right) \cdot \nabla u_{\lambda}+\lambda a e^{2 \sqrt{\lambda} \rho} \eta^{2} u_{\lambda}^{2}\right) \mathrm{d} x \mathrm{~d} t \\
& -\int_{A_{\varepsilon} \backslash \overline{A_{2 \varepsilon}}} e^{2 \sqrt{\lambda} \rho} u_{\lambda} \eta \nabla \eta \cdot \nabla u_{\lambda} \mathrm{d} x \mathrm{~d} t+\int_{A_{\varepsilon} \backslash \overline{A_{2 \varepsilon}}} \nabla\left(e^{2 \sqrt{\lambda} \rho} \eta u_{\lambda}\right) \cdot u_{\lambda} \nabla \eta \mathrm{d} x \mathrm{~d} t .
\end{aligned}
$$

Due to estimate (5.1), there exists a constant $C>0$ such that

$$
\left\|u_{\lambda}\right\|_{L^{2}\left(0, T ; H_{0}^{1}(\Omega)\right)} \leq C\left(\left\|f_{\lambda}\right\|_{L^{2}\left(Q_{T}\right)}+\left\|g_{\lambda}\right\|_{L^{2}(\Omega)}\right) .
$$

This yields

$$
\begin{aligned}
& \left|\int_{A_{\varepsilon} \backslash \overline{A_{2 \varepsilon}}} e^{2 \sqrt{\lambda} \rho} u_{\lambda} \eta \nabla \eta \cdot \nabla u_{\lambda} \mathrm{d} x \mathrm{~d} t\right| \\
& \quad \leq C e^{2 \sqrt{\lambda} M}\|\nabla \eta\|_{\infty}\left(\left\|f_{\lambda}\right\|_{L^{2}\left(Q_{T}\right)}^{2}+\left\|g_{\lambda}\right\|_{L^{2}(\Omega)}^{2}\right)
\end{aligned}
$$


where $M$ is defined by

$$
M:=\sup _{x \in Q_{T} \backslash A_{2 \varepsilon}} \rho(x, t) .
$$

Using integration by parts in the space variable, we also have

$$
\begin{aligned}
& \left|\int_{A_{\varepsilon} \backslash \overline{A_{2 \varepsilon}}} \nabla\left(e^{2 \sqrt{\lambda} \rho} \eta u_{\lambda}\right) u_{\lambda} \cdot \nabla \eta \mathrm{d} x \mathrm{~d} t\right| \\
& \quad \leq C e^{2 \sqrt{\lambda} M}\left(\|\nabla \eta\|_{\infty}+\|\Delta \eta\|_{\infty}\right)\left(\left\|f_{\lambda}\right\|_{L^{2}\left(Q_{T}\right)}^{2}+\left\|g_{\lambda}\right\|_{L^{2}(\Omega)}^{2}\right) .
\end{aligned}
$$

To treat the last term in the right-hand side of inequality (6.7), we simply decompose

$$
\begin{aligned}
& \int_{Q_{T}} e^{2 \sqrt{\lambda} \rho} \eta u_{\lambda} \partial_{t}\left(\eta u_{\lambda}\right) \mathrm{d} x \mathrm{~d} t \\
& \quad=\int_{A_{\varepsilon}} e^{2 \sqrt{\lambda} \rho} \eta^{2} u_{\lambda} \partial_{t} u_{\lambda} \mathrm{d} x \mathrm{~d} t+\int_{A_{\varepsilon}} e^{2 \sqrt{\lambda} \rho} \eta u_{\lambda}^{2} \partial_{t} \eta \mathrm{d} x \mathrm{~d} t
\end{aligned}
$$

and use the upper bound

$$
\left|\int_{A_{\varepsilon}} e^{2 \sqrt{\lambda} \rho} \eta u_{\lambda}^{2} \partial_{t} \eta \mathrm{d} x \mathrm{~d} t\right| \leq C e^{2 \sqrt{\lambda} M}\left(\left\|\partial_{t} \eta\right\|_{\infty}\right)\left(\left\|f_{\lambda}\right\|_{L^{2}\left(Q_{T}\right)}^{2}+\left\|g_{\lambda}\right\|_{L^{2}(\Omega)}^{2}\right) .
$$

Coming back to (6.7), and using (6.8)-(6.10), we deduce:

$$
\begin{aligned}
& \frac{\lambda \delta}{4} \int_{Q_{T}} e^{2 \sqrt{\lambda} \rho} \eta^{2} u_{\lambda}^{2} \mathrm{~d} x \mathrm{~d} t \\
& \quad \leq C e^{2 \sqrt{\lambda} M}\left(\|\nabla \eta\|_{\infty}+\|\Delta \eta\|_{\infty}+\left\|\partial_{t} \eta\right\|_{\infty}\right)\left(\left\|f_{\lambda}\right\|_{L^{2}\left(Q_{T}\right)}^{2}+\left\|g_{\lambda}\right\|_{L^{2}(\Omega)}^{2}\right) \\
& \quad+\int_{A_{\varepsilon}}\left[\nabla\left(e^{2 \sqrt{\lambda} \rho} \eta^{2} u_{\lambda}\right) \cdot \nabla u_{\lambda}+\lambda a e^{2 \sqrt{\lambda} \rho} \eta^{2} u_{\lambda}^{2}+e^{2 \sqrt{\lambda} \rho} \eta^{2} u_{\lambda} \partial_{t} u_{\lambda}\right] \mathrm{d} x \mathrm{~d} t .
\end{aligned}
$$

Since the function $e^{2 \sqrt{\lambda} \rho} \eta^{2} u_{\lambda}$ is in $L^{2}\left(0, T ; H_{0}^{1}(\Omega)\right)$, it is an admissible test function for problem $\left(P_{\lambda}\right)$, and since $\eta$ is identically null outside $A_{\varepsilon}$, there holds

$$
\int_{A_{\varepsilon}}\left[\nabla\left(e^{2 \sqrt{\lambda} \rho} \eta^{2} u_{\lambda}\right) \cdot \nabla u_{\lambda}+\lambda a e^{2 \sqrt{\lambda} \rho} \eta^{2} u_{\lambda}^{2}+e^{2 \sqrt{\lambda} \rho} \eta^{2} u_{\lambda} \partial_{t} u_{\lambda}\right] \mathrm{d} x \mathrm{~d} t=\int_{A_{\varepsilon}} e^{2 \sqrt{\lambda} \rho} \eta^{2} u_{\lambda} f_{\lambda} \mathrm{d} x \mathrm{~d} t
$$

which implies that

$$
\frac{\lambda \delta}{4} \int_{Q_{T}} e^{2 \sqrt{\lambda} \rho} \eta^{2} u_{\lambda}^{2} \mathrm{~d} x \mathrm{~d} t \leq C e^{2 \sqrt{\lambda} M}\left(\left\|f_{\lambda}\right\|_{L^{2}\left(Q_{T}\right)}^{2}+\left\|g_{\lambda}\right\|_{L^{2}(\Omega)}^{2}\right)+\int_{A_{\varepsilon}} e^{2 \sqrt{\lambda} \rho} \eta^{2} u_{\lambda} f_{\lambda} \mathrm{d} x \mathrm{~d} t
$$

where $C$ depends on $\varepsilon,\|\nabla \eta\|_{\infty},\|\Delta \eta\|_{\infty}$ and $\left\|\partial_{t} \eta\right\|_{\infty}$.

Now we take the particular choice

$$
\rho(x, t):=\min \left\{\frac{\delta}{2}, \sqrt{\frac{\delta}{2}}\right\} \operatorname{dist}\left((x, t), A_{2 \varepsilon}^{c}\right),
$$


which satisfies all our needed assumptions (i.e. $\rho$ is Lipschitz with $\max \left\{\left|\partial_{t} \rho\right|,|\nabla \rho|^{2}\right\} \leq \delta / 2$ and supported in $\left.A_{\varepsilon}\right)$. In this case, $M=0$ so that (6.11) reduces to

$$
\frac{\lambda \delta}{4} \int_{A_{\varepsilon}} e^{2 \sqrt{\lambda} \rho} \eta^{2} u_{\lambda}^{2} \mathrm{~d} x \mathrm{~d} t \leq C+\int_{A_{\varepsilon}} e^{2 \sqrt{\lambda} \rho} \eta^{2} u_{\lambda} f_{\lambda} \mathrm{d} x \mathrm{~d} t
$$

and hence

$$
\int_{A_{\varepsilon}} e^{2 \sqrt{\lambda} \rho} \eta^{2} u_{\lambda}(x)\left(\frac{\lambda \delta}{4} u_{\lambda}-f_{\lambda}\right) \mathrm{d} x \leq C
$$

We end this section by noticing that Theorem 1.3 follows directly from Lemma 6.3.

Corollary 6.4. In the particular case when $f=0$ in $Q_{T} \backslash O_{a}$ we get the useful rate of convergence of $u_{\lambda} \rightarrow 0$ as $\lambda \rightarrow \infty$ far from $O_{a}$ :

$$
\lambda e^{c_{\delta} \varepsilon \sqrt{\lambda}} \int_{A_{2 \varepsilon}} u_{\lambda}^{2} \mathrm{~d} x \mathrm{~d} t \leq C
$$

\section{REFERENCES}

[1] P. Álvarez-Caudevilla and A. Lemenant, Asymptotic analysis for some linear eigenvalue problems via Gamma-convergence. Adv. Differ. Equ. 15 (2010) 649-688.

[2] P. Álvarez-Caudevilla and J. López-Gómez, Semiclassical analysis for highly degenerate potentials. Bull. Am. Math. Soc. 136 (2008) 665-675.

[3] I. Antón and J. López-Gómez, The maximum principle for cooperative periodic-parabolic systems and the existence of principle eigenvalues, in World Congress of Nonlinear Analysts '92 (Tampa, FL, 1992). de Gruyter, Berlin (1996) 323-334.

[4] L. Boudin, C. Grandmont and A. Moussa, Global existence of solutions to the incompressible Navier-Stokes-Vlasov equations in a time-dependent domain. J. Differ. Equ. 262 (2017) 1317-1340.

[5] R.M. Brown, W. Hu and G.M. Lieberman, Weak solutions of parabolic equations in non-cylindrical domains. Proc. Am. Math. Soc. 125 (1997) 1785-1792.

[6] S.-S. Byun and L. Wang, Parabolic equations in time dependent Reifenberg domains. Adv. Math. 212 (2007) $797-818$.

[7] J. Calvo, M. Novaga and G. Orlandi, Parabolic equations in time dependent domains. J. Evol. Eqs. 17 (2017) $781-804$.

[8] D. Daners and C. Thornett, Periodic-parabolic eigenvalue problems with a large parameter and degeneration. J. Differ. Equ. 261 (2016) 273-295.

[9] Y. Du and R. Peng, The periodic logistic equation with spatial and temporal degeneracies. Trans. Am. Math. Soc. 364 (2012) 6039-6070.

[10] L.C. Evans, Partial Differential Equations. Graduate Studies in Mathematics. American Mathematical Society, RI (1998).

[11] J. García-Melián, R. Gómez-Reñasco, J. López-Gómez and J.C. Sabina de Lis, Point-wise growth and uniqueness of positive solutions for a class of sublinear elliptic problems where bifurcation from infinity occurs. Arch. Ration. Mech. Anal. 145 (1998) 261-289.

[12] P. Hess, Periodic-Parabolic Boundary Value Problems and Positivity. Vol. 247 of Pitman Research Notes in Mathematics. Longman Scientific and Technical, Harlow (1991).

[13] G. Savaré, Parabolic problems with mixed variable lateral conditions: an abstract approach. J. Math. Pures Appl. 76 (1997) 321-351.

[14] B. Simon, Semiclassical analysis of low lying eigenvalues. II. Tunneling. Ann. Math. 120 (1984) 89-118. 